\title{
Background Information Detection in Substations Based on Anomaly Detection
}

\author{
Ge Li-Qing, Wang Jian-Feng, Teng Jing-Yu, Yang Ming \\ NR Electric Co., Ltd, NanJing, 211102 China
}

\begin{abstract}
The substations are important parts of modern electrical grids. In this sense, it is necessary to detect the anomaly and problems in it. In this paper, we study on the information detection in substations based on traditional anomaly detection algorithms. The data flow from the background information is represented by feature vectors. And those from the historical data are used to build the background references. Afterward, the feature vector of the input data flow is examined using the anomaly detection algorithm. Based on the results, the anomaly in the background information in the substations can be found and located. Then, some high-precision identification algorithms can be further employed to recognition the type of the problems. In this way, the problems occurred in the substations can be found and solved in time.
\end{abstract}

\section{Introduction}

The safety of modern electric grids is closely related to people's daily life. As an important part of the electrical system, the protection of substations are important tasks to manage the smooth operating the whole system [1-3]. Many previous works focused on the protection of substations using measures including backup protection, logic visualization, fault analysis, etc. [4-12]. With a large amount of information in the substations, the states and conditions can be inferred or judged from these data. When there are anomalies occurred, the system should immediately make responses and send the alarms to the works. Therefore, it is necessary to research on the information detection algorithms about the substations. In this way, the occurred problems can be found and handled in time to avoid the corruption of the whole system. To some extent, the previous fault analysis or diagnose did this job but it focused on the faults. In fact, there many much more anomalies in the substation information while should also be considered [13-16].

In this paper, we research on the background information detection method in substations based on traditional anomaly detection algorithms. The anomaly detection tends to find the information with great differences with the background. It is a typical unsupervised classification representative and can be implemented with high efficiency. In the previous works, the anomaly detection algorithms were successfully applied to image processing, target detection, etc. [17-19]. This paper applies anomaly detection techniques into the information detection in substations. For each data flow from the background information, it is first converted to feature vectors. Then, it is compared with the background feature vectors and the divergence is calculated. When the differences are higher than a preset threshold, the feature vector is judged to be an anomaly one and the corresponding data flow is assumed to be an anomaly one. Afterwards, more precise measures can be taken to recognize the types of the anomaly and the workers can deal with the problems. With the help of anomaly detection, the problems occurred in the substations can be found and located in time. Then, corresponding measures can be taken to deal with these problems.

\section{Basic Theory of Anomaly Detection}

Anomaly detection can be seen as a binary classification problem, in which the input is judged to be a normal or anomaly sample. The mathematical model is formulated as a binary hypothesis test given as follow:

$$
\begin{aligned}
& \text { 主 } H_{0}: r=n \\
& \frac{1}{1} H_{1}: r=s+n
\end{aligned}
$$

In Equation (1), the hypothesis $H_{0}$ indicates there is no anomaly and the hypothesis $H_{1}$ indicates there is an anomaly signal $s$. Here, $n$ represents the background, which provides references for anomaly detection. The background reflects the properties of most of the data flows. When a new input has notable differences with the background, it is assumed to be an anomaly one. Generally, the binary hypothesis test in Equation (1) can be solved in a statistical way. At first, the statistical distribution of the background can be modelled based on the approaching of large amount of data. Afterwards, the new input is fitted with the statistical model and calculate the residual. When the residual is large enough (usually compared with a threshold $T$ ), the input is judged to be 
anomaly. On the contrary, it is assumed to share similar properties with the background. So, in this situation, it is judged not an anomaly.

With the development in previous years, the anomaly detection algorithms became more and more stable. Many new proposed technologies were also used in it such as sparse representation, deep learning models. It is assumed that the anomaly detection method could operate with high efficiency to find and locate the abnormal samples in the large amount of data flows. For the background information in the substations, they are important in the monitoring of the whole system. So, the anomaly occurred in them should be carefully considered. For the implementation of the anomaly detection algorithms, the historical data in the substations could provide the background references in Equation (1). So, for the test sample, it can be processed and judged whether it is an anomaly or not. In this sense, the traditional anomaly detection algorithms can be effectiveness used in the information detection in substations.

\section{Application of Anomaly Detection in Information Detection in Substations}

In this paper, we apply traditional anomaly detection algorithms into information detection in substations. The main objective is to quickly locate the potential threats in the dataflows from the background information in substations. The basic procedure is illustrated as Fig .1, which can be summarized as following steps:

Step 1: Feature generation: the original data flows in the substations may have high dimensions. In order to enhance the detection efficiency, some feature generations methods can be employed to reduce them to low dimensions.

Step 2: Construction of the background feature vectors: based on the large amount of historical data, their feature vectors are generated to construct the background references for the anomaly detection algorithms.

Step 3: Detection of the anomaly for a new input: for a new input, its feature vector is first generated as Step 1. Then, the anomaly detection algorithm is performed on it based on the background references from Step 2.

After the information detection, a certain input is judged to be a normal one or anomaly. Once an anomaly is reported, the system further classifies it as a certain type of threats. Then, the system could guide the works to handle these problems.

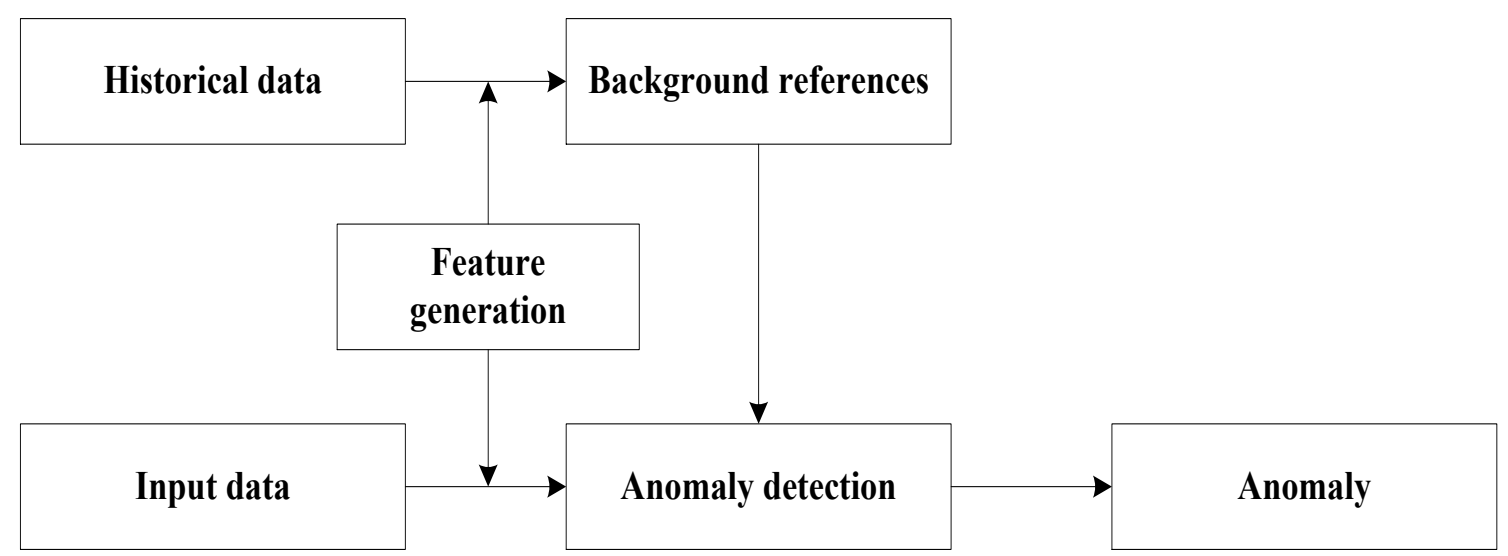

Fig. 1 Basic procedure of applying anomaly detection algorithm into information detection in the substations.

\section{Conclusion}

In this paper, we research on the information detection in substations based on the anomaly detection algorithms. The background references are first established based on the historical data in the substations using some feature generation methods. For the input data flow, it is first converted into the feature vector and then processed by the anomaly detection method. And the results will report whether it is an anomaly. Based on the information detection, the problems occurred in the substations can be quickly located for the further identification. Consequently, the workers can take measures according to the reported alarms to fix the problems. So, using these technologies, the substations can be more robust. In the future researches, we will continue the study on the high-precision identification methods of the anomaly occurred in the substations. Using these methods, the detailed type of the anomaly can be confirmed to better help the workers to solve the problems.

\section{Acknowledgement}

This work was supported by Technology Funding Project of State Grid Corporation of China (Grant No. 52153218000G).

\section{References}

1. Borlea I, Buta A and Dusa et al. 2005 DIASE-Expert system fault diagnosis for Timisoara $200 \mathrm{kV}$ Substation in Proc. EUROCON pp 221-224. 
2. Wang Y and Pan Z 2011 Study on test technology of smart substation secondary system in Proc. International Conference on Consumer Electronics, Communications and Networks pp 1-4.

3. Kezunovic M 2009 Substation fault analysis requirement in Proc. IEEE Power \& Energy Society General Meeting pp 1-2.784-787

4. Lian J, Li H and Song K 2011 The analysis and corrective measures of common problems of $10 \mathrm{kV}$ capacitor bank in substation in Proc International Conference on Advanced Power System Automation and Protection pp 1750-1752

5. Gogan P L and Wyckoff G D 2012 Design and construction of sustainable substations in Proc. PES T\&D pp 1-4.

6. Li Q, Zhou Z and Du D, et al. 2013 A novel substation area backup protection for smart substation in Proc. IEEE PES Asia-Pacific Power and Energy Engineering Conference pp 1-4.

7. Wang Z, Jin $\mathrm{N}$ and Zhang J, et al. 2014 Fault information and diagnosis modelling of on-line communication monitoring system for digital substation in Proc. IEEE PES General Meeting | Conference \& Exposition pp 1-5.

8. Liu Y, Gao H and Gao W, et al. 2017 Development of a substation-area backup protective relay for smart substation IEEE Transactions on Smart Grid vol 8 no 6 pp 2544-2553.

9. Zhang H, Dou R and Zhi M, et al. 2016 The technology of interlocking logic visualization for smart substation in Proc. China International Conference on Electricity Distribution pp 1-4.

10. Dehbonei H 2016 Earthing-Substation fire and station transformer customers supply transfer voltage issue in Proc. Down to Earth Conference pp 1-7.

11. Vardhan H and Ramlachan R 2018 Deploying digital substations: Experience with a digital substation pilot in north America in Proc. Annual Conference for Protective Relay Engineers pp 1-9.
12. Li Z and Ma Q 2018 Discussion on optimization measures of relay protection technology in intelligent substation in Proc. IEEE Advanced Information Management, Communicates, Electronic and Automation Control Conference pp 1593-1596.

13. Topolsky D V, ToPolskaya I G and Topolsky N D 2018 Development of an intelligent measuring system for digital substations in Proc. pp International Multi-Conference on Industrial Engineering and Modern Technologies 1-4.

14. Nassu B T, Lourival L and Bruno M et al. 2018 Image-based state recognition for disconnect switches in electric power distribution substations in Proc. SIBGRAPI Conference on Graphics, Patterns and Images pp 432-439.

15. Wang H, Li J and Zhou Y, et al. 2019 Research on the technology of indoor and outdoor integration robot inspection in substation in Proc. IEEE 3rd Information Technology, Networking, Electronic and Automation Control Conference pp 2366-2369.

16. Nichani A M and Swarup S 2018 Modelling and simulation of digital substation automation for intersubstation line protection in Proc. National Power Systems Conference pp 1-6.

17. Li Y, Wang J and Liu X, et al. 2018 DIM Moving Target Detection using Spatio-Temporal Anomaly Detection for Hyperspectral Image Sequences IEEE International Geoscience and Remote Sensing Symposium pp 1-4.

18. Ma N, Peng Y and Wang S, et al. 2017 A weight SAE based hyperspectral image anomaly targets detection IEEE International Conference on Electronic Measurement \& Instruments pp 1-4.

19. Ning H, Zhang $X$ and Zhou H, et al. 2019 Hyperspectral Anomaly Detection via Background and Potential Anomaly Dictionaries Construction IEEE Transactions on Geoscience and Remote Sensing vol 57 no 4 pp 2263-2276. 